\title{
House dust mite-induced endoplasmic reticulum stress mediates mucus MUC5AC hyper secretion via TBK1-STAT6/NF-xB in asthmatic mice
}

Yun Zhang ${ }^{1}$, Hongmei Tang ${ }^{1}$, Xiefang Yuan ${ }^{1}$, ning ma ${ }^{1}$, Hang $\mathrm{Hu}^{1}$, Xiaoyun Wang ${ }^{1}$, Chunfeng Liu ${ }^{1}$, Guofeng $\mathrm{Xu}^{1}$, Yuejiao $\mathrm{Li}^{1}$, Songping Wang ${ }^{1}$, Linlin Guo ${ }^{2}$, Jun Deng ${ }^{1}$, and Xing Wang ${ }^{1}$

${ }^{1}$ The Affiliated Hospital of Southwest Medical University

${ }^{2}$ The Ohio State University Wexner Medical Center

September 23, 2020

\begin{abstract}
ABSTRACT Background Exposed to house dust mite (HDM) is known to be associated with allergic asthma. Endoplasmic reticulum (ER) stress is involved in the regulation of mucus hyper secretion. However, the mechanism remains unclear. Our main goal was to investigate the relationship of TBK1 pathway regulates the expression of MUC5AC under the HDM induced ER stress promote allergic asthma. Methods Using HDM induced allergic mice and stimulated over expression of MUC5AC in human airway epithelial cells. We examined the mucus and the expression of ER stress markers both in vivo and in vitro. Additionally, we investigated whether TBK1, NF- $\nsim$ B, STAT6 play an important role in the HDM induced ER stress promote airway mucus hyper secretion. Results Mice exposed to HDM were identified ER stress, hyper secretion of mucus, and activated TBK1-NF- $x$ B/STAT6 signaling pathway in airway epithelial cells of asthmatic mice. Similarly results had also been observed in the human airway epithelial cells after exposed to HDM. Both in vivo and in vitro study not only revealed that an antiallergy drug, Amlexanox, reduced super response of mucus and weaken TBK1-NF- $\varkappa$ B/STAT6 signal of induced asthma. But also indicated HDM induced ER stress result in over production of MUC5AC, which can be decreased by the inhibition of TBK1, NF- $\varkappa$ B, STAT6, or even by using ER stress inhibitor 4-PBA, respectively. Conclusions Our results show that TBK1$\mathrm{NF}-\varkappa \mathrm{B} / \mathrm{STAT} 6$ plays a pivotal role in the HDM induced ER stress result in over production of mucus protein MUC5AC in the asthmatic airway.
\end{abstract}

\section{Hosted file}

1--Complete Manuscript with Figures and supplemental for ALLERGY.pdf available at https:// authorea.com/users/361307/articles/482747-house-dust-mite-induced-endoplasmic-reticulumstress-mediates-mucus-muc5ac-hyper-secretion-via-tbk1-stat6-nf-\%CE\%BAb-in-asthmatic-mice 